\title{
Heartbeat: Is atrial fibrillation ablation effective in patients with hypertrophic cardiomyopathy?
}

Atrial fibrillation (AF) is a common co-morbidity in patients with hypertrophic cardiomyopathy (HCM) and it can contribute to poor outcomes. Therefore, identification of safe and effective strategies for controlling AF in HCM are of substantial interest to researchers, clinicians and patients.

In this issue of Heart, Providencia and colleagues (see page 1533) report the findings from a systematic review and meta-analysis of published literature which takes a closer look at the safety and efficacy of AF ablation in HCM. The authors identify 15 potentially relevant studies and pool data from 4 cohort studies to compare the rates of success and complications after $\mathrm{AF}$ ablation in patients with HCM with those without HCM. They showed that people with HCM are about 2 times more likely to have a relapse after a single ablation therapy than those without HCM (OR 2.25, CI 1.09 to 4.64 ) (figure 1 ). They further note that HCM patients are more likely to be prescribed anti-arrhythmic drugs but overall the risk of adverse events after ablation therapy seems low. In exploratory subgroup analyses, they generate the hypothesis that the differences in success rates after ablation therapy between the HCM and non-HCM patients may be due to larger atrial size and/or higher rate of persistent $\mathrm{AF}$ in HCM than those without HCM.

In an accompanying editorial, Prutkin and Owens (see page 1513) remind us of the challenges and limitations of pharmacological rhythm control in patients with HCM and mention why in this context interventional approaches are likely to be a very attractive option. They further stress the limited data available to date to be able to make robust clinical recommendations for this option. In particular, they note the absence of randomised trials and highlight the fact that some of the observational studies are now several years old, which could at least have an impact on the absolute relapse rates reported in this meta-analysis. Despite these limitations,

Correspondence to Professor Catherine M Otto, Division of Cardiology, University of Washington, Seattle, WA 98195, USA; cmotto@u.washington.edu

\section{Freedom from AF/AT relapse after a single procedure}

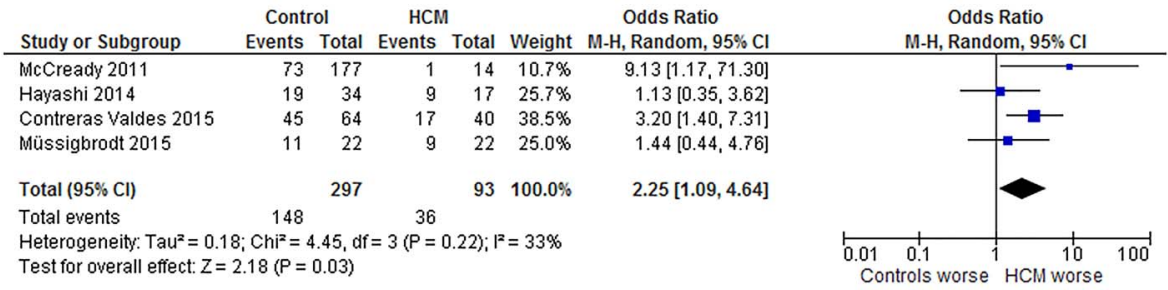

\section{Freedom from AF/AT relapse after one or more procedures}

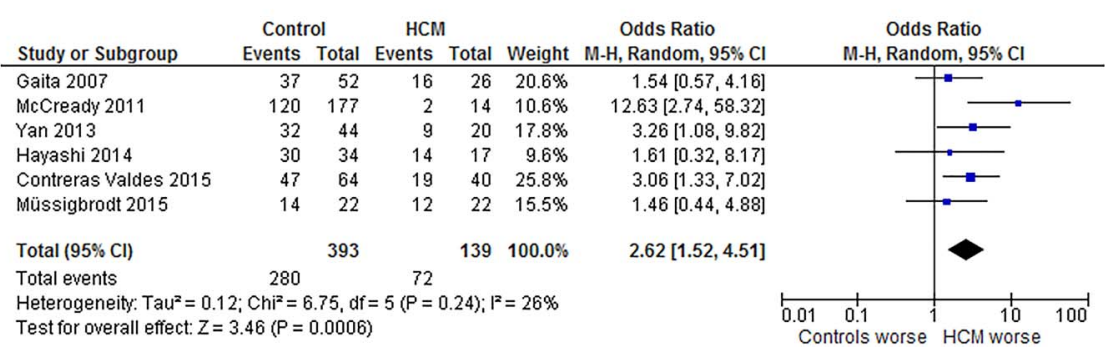

Figure 1 Forest plots comparing procedural outcomes (freedom from AF/AT relapse) of catheter ablation of $A F$ in patients with and without $H C M$. AF, atrial fibrillation; $A T$, atrial tachycardia; HCM, hypertrophic cardiomyopathy.

they acknowledge that "HCM patients fare worse - lower success rate, more repeat procedures, and greater need for antiarrhythmic therapy - with AF ablation than those of the general population" and share their insights as to why this may be the case and how we could tackle this issue in the future.

Between 35 and $50 \%$ of patients with Trisomy 21 (Down syndrome, DS) have associated congenital heart disease, particularly atrioventricular septal defects and ventricular septal defects that are likely to result in equalization of systemic and pulmonary pressures or Eisenmenger syndrome (ES) unless corrective procedures are performed early in life. Over the past 50 years, the management of Down syndrome (DS) patients has evolved with more receiving corrective therapy within the first year of life and fewer developing ES. In a study of 894 DS patients at risk of ES, Körten and colleagues (see page 1552) confirmed a higher mortality rate in those who developed ES compared to those without ES (HR 18.1; 95\% CI 7.2 to $45.4 ; \mathrm{p}<0.0001)$. However, overall survival (figure 2) for entire DS cohort continues to show excess mortality compared to expected survival.

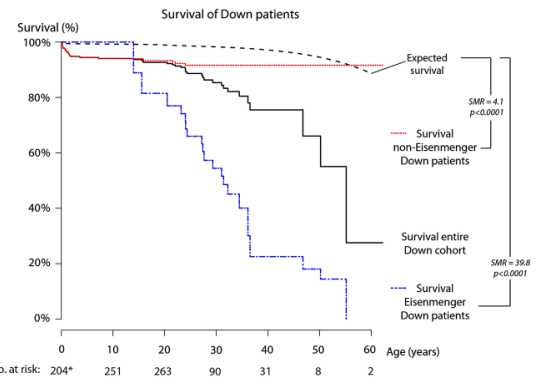

Figure 2 Survival in Down syndrome by time-dependent survival analysis stratified by the presence of Eisenmenger syndrome and compared with the expected survival of an age-matched and gender-matched sample of the general population. SMR, standardised mortality rate. *Number at risk provided for the first year of life as well as 10,20,30, 40, 50 and 60 years of age.

In the accompanying editorial, Dimopoulos and Kempny (see page 1515) remind us that DS patients require periodic cardiac evaluation, even after a corrective procedure, because some still will develop severe pulmonary hypertension and some have associated conditions, such as atrioventricular valve regurgitation. Management of DS patients often is complicated by limited understanding of the 
need for medical follow-up, poor compliance with medical therapy and inadequate support systems. In addition, as DS patients age, atherosclerotic risk factors are prevalent including obesity, diabetes, hypothyroidism, obstructive sleep apnea and elevated plasma lipid levels. The authors suggest that optimal care of DS patients "can only be achieved with a multidisciplinary approach and team effort, including patients, their families and carers, physicians and healthcare workers of different expertise, and patient associations. Active screening for longterm sequelae of $\mathrm{CHD}$ and other comorbidities is paramount, while promoting a healthy lifestyle and proactive control of cardiovascular risk factors.”

All health care providers agree that we should strive to provide high quality care to all our patients. We disagree, however, on how to measure quality of care and how to present this information to the public. In a provocative editorial, Ray, Nishimura, Clarke and Simpson discuss the use of reliable metrics for clinical performance with identification of outliers as one approach to this issue (see page 1518). They also emphasize the role of clinical culture to ensure acceptance of this approach by health care providers and the key concept that metrics should be "used appropriately as a tool for both quality assurance and quality improvement, not as a competitive league table or as a means to castigate clinicians who are actually performing at a high level." Importantly, they argue that institutional commitment with a "supportive and transparent culture" is essential because individual health care providers do not work in isolation (figure 3). "Excellent clinical services have structures in place that seek continuous feedback from service users and which can identify and deal with potential performance problems at an early stage and so avoid getting to the point where patient safety is at risk."

The Education in Heart article in this issue (see page 1589) summarizes the clinical utility of cardiac magnetic resonance imaging (CMR) for the general cardiologist with nice examples of typical findings (figure 4). As with all Education in Heart articles, CPD/CME credits are available for this article and the accompanying multiple choice questions on our online website.

Readers will also want to read the excellent review article "Approach to

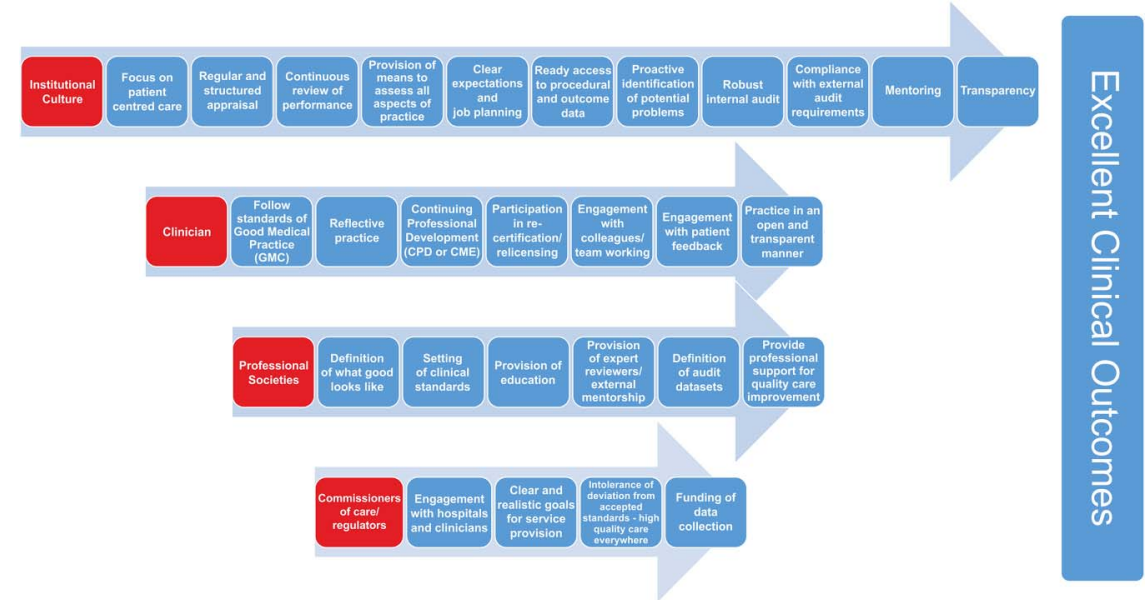

Figure 3 Working towards clinical excellence.

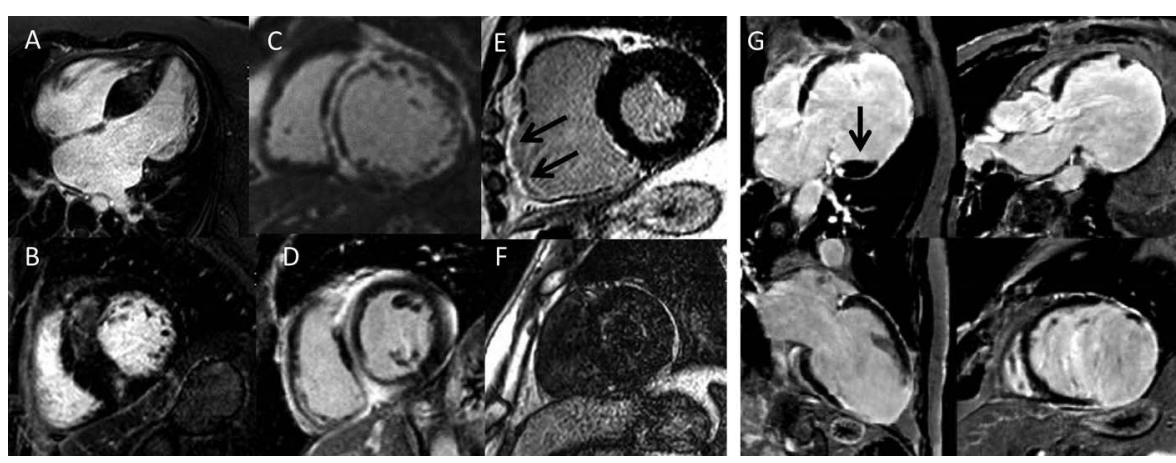

Figure 4 CMR in Cardiomyopathy. (A) Hypertrophic cardiomyopathy with severe asymmetrical septal hypertrophy and fibrosis on 4-chamber late gadolinium enhancement (LGE) image.

(B) Short axis view of the same HCM patient demonstrating the marked diffuse septal LGE.

(C) Dilated non-ischaemic cardiomyopathy with extensive mid-wall fibrosis. (D) Patient presenting with chest pain with extensive septal mid-wall and epicardial lateral wall fibrosis: CMR findings typical of myocarditis. (E) 4-chamber LGE image showing dilated right ventricle with RV late enhancement (arrows). Cine imaging also demonstrated impaired function and dyskinetic regional wall motion (Video 5) consistent with a diagnosis of arrhythmogenic right ventricular cardiomyopathy). (F) Inability to null the LV myocardium during LGE imaging which is a classical CMR feature of cardiac amyloid. (G) Panel of 4 LGE images demonstrating an ischaemic cardiomyopathy with severely dilated LV and full thickness myocardial infarction (white) and an aneurysmal lateral wall. There is also a basal lateral thrombus (see arrow).

Residual Pulmonary Valve Dysfunction in Adults with Repaired Tetralogy of Fallot" by Dr. Yuli Y Kim (see page 1520) given the increasing number of patients with surviving to adulthood with this condition. Clinical cardiologists can expect to see more of these patients in their practices so we need to be aware of potential long term complications and indications for referral to a center that specializes in adult congenital heart disease.

The Image Challenge in this issue is "Contemporary imaging following atrial redirection surgery for transposition of the great arteries" (see page 1551) with interesting echocardiographic and CMR images.

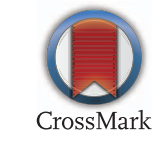

To cite Rahimi K, Otto CM. Heart 2016;102:15111512.

Heart 2016;102:1511-1512.

doi:10.1136/heartjnl-2016-310468 